\title{
Non-Translation-Invariant States in Two Dimensions
}

\author{
S. B. Shlosman \\ Institute for Problems of Information Transmission, Moscow, USSR
}

\begin{abstract}
We construct a class of models with translation-invariant interaction for which in dimension two there already exist non-periodic Gibbs states at low temperatures.
\end{abstract}

\section{Introduction}

The hypothesis about absence of non-translation-invariant states in the twodimensional Ising model was one of the most long-standing problems. The first major development in this direction was made by Gallavotti [1], who proved, by means of virial expansion, that at low temperature the ( \pm -boundary condition leads to a translation-invariant state. He showed that the separation line between $(+)$-phase and ( - )-phase in a volume $V$ with such a boundary condition fluctuates at non-zero temperature with a fluctuation of order $\sqrt[4]{V}$. Afterwards, several papers were published on this subject, until Aizenman [2] proved the validity of the above hypothesis. This result can be stated in the following way: All nonperiodic ground states of the two-dimensional Ising model are unstable.

In 1980, R. L. Dobrushin conjectured that the same instability holds (at low temperatures, at least)for any two-dimensional model whose hamiltonian has only finitely many periodic ground states (pure phases) and satisfies the Peierls condition [3]: i.e., the creation of an "island" $D$ of one pure phase in a "sea" of another pure phase leads to an increase of energy which is greater than $\varrho \cdot|\partial \mathscr{D}|$, where $\varrho$ is some positive constant (the "Peierls constant"), and $|\partial \mathscr{D}|$ is the length of the boundary of $\mathscr{D}$.

While the proof of this conjecture is still in progress, it was surprising to find a class of models which possesses both periodic and non-periodic Gibbs states at a cost of having infinitely many periodic ground states. The Peierls condition is still satisfied. The underlying non-translation invariant ground states are of the following "stair" structure:

$$
\varphi_{\left(t_{1}, t_{2}\right)}=n t_{1}, \quad\left(t_{1}, t_{2}\right) \in \mathbb{Z}^{2}, \quad n \in \mathbb{Z}^{1}, \quad n \neq 0 .
$$


This ground state is stable for the following reason: While in the Ising model the corresponding $( \pm)$ ground state has the form

$$
\varphi_{\left(t_{1}, t_{2}\right)}= \begin{cases}0 & t_{1} \leqq 0 \\ 1 & t_{1}>0\end{cases}
$$

with only one contour to separate different phases, which is thus free to fluctuate, in our model the ground state under discussion has infinitely many contours, which cannot fluctuate so freely due to interacting with each other. The precise meaning of this argument is given in the next section.

\section{The Main Result}

Let $\mathbb{Z}^{2}$ be, as usual, a two-dimensional lattice, and for any $t=\left(t_{1}, t_{2}\right)$ a spin variable $\varphi_{t}$ is given with values in $\mathbb{Z}^{1}$. The interaction is defined through the function $U(n)$, $n \in \mathbb{Z}^{1}$, which is supposed to be

(i) Convex: $2 U(n)<U(n+k)+U(n-k), n, k \in \mathbb{Z}^{1}, k>0$,

(ii) Symmetric: $U(n)=U(-n)$.

The (formal) hamiltonian $H$ of a configuration $\varphi=\left\{\varphi_{t}\right\}$ is given by the formula

$$
H\left(\varphi_{t}\right)=(1 / 2) \sum_{s, t \in \mathbb{Z}^{2},|s-t|=1} U\left(\varphi_{s}-\varphi_{t}\right)
$$

A configuration $\varphi$ is called a ground state if for any other configuration $\varphi^{\prime}$ which coincides with $\varphi$ almost everywhere the (well-defined) difference $H\left(\varphi^{\prime}\right)-H(\varphi)$ is strictly positive. One sees immediately that any constant configuration

$$
\varphi_{t} \equiv n \in \mathbb{Z}^{1}
$$

forms a ground state. So our model indeed has infinitely many periodic ground states. There are also non-periodic ground states of the hamiltonian (1), for example,

$$
\varphi_{t}=\left\{\begin{array}{ll}
1 & t_{1}>0 \\
0 & t_{1} \leqq 0
\end{array} \quad t=\left(t_{1}, t_{2}\right)\right.
$$

If one defines the mean energy of a configuration $\varphi$ by the formula

$$
h(\varphi)=\lim _{\Lambda \rightarrow \infty} \frac{1}{|\Lambda|} \sum_{s, t \in \Lambda,|s-t|=1} U\left(\varphi_{s}-\varphi_{t}\right)
$$

(when the limit exists), then one finds that the mean energy of all periodic ground states (2) is, evidently, the same, and it coincides with that of a non-periodic ground state of type (3).

Our interest in the model under discussion is based on the observation that the hamiltonian (1) also possesses a class of ground states $\left\{\Psi^{n, k, i}\right\} n, k \in \mathbb{Z}^{1}, i=1,2$ given by

$$
\Psi_{t}^{n, k, i}=n t_{i}+k
$$


This class has two interesting features:

1) $h\left(\Psi^{h_{1}, k_{1}, i_{1}}\right)>h\left(\Psi^{h_{2}, k_{2}, i_{2}}\right)$, if $\left|n_{1}\right|>\left|n_{2}\right|$; in particular, if the ground state $\Psi^{n, k, i}$ is non-periodic $(n \neq 0)$, then its mean energy exceeds that of a constant ground state;

2) each ground state $\Psi^{n, k, i}$ is stable in the sense that for each $n, k, i$ there exists a Gibbs state of the hamiltonian (1) at low temperature which is a small perturbation of the corresponding ground state.

To make our statements precise, let $\Lambda \subset \mathbb{Z}^{2}$ be a finite region, and consider the set of configurations $\Phi_{\Lambda}^{n, k, i}=\left\{\varphi_{s}, s \in \mathbb{Z}^{2}, \varphi_{s}=\Psi_{s}^{n, k, i}, s \notin \Lambda\right\}$. The hamiltonian $H$ in $\Lambda$ with boundary conditions $\Psi^{n, k, i}$ is defined in the following way:

$$
H^{n, k, i}(\varphi)=(1 / 2) \sum_{\substack{s, t \in \Lambda \\|s-t|=1}} U\left(\varphi_{s}-\varphi_{t}\right)+\sum_{\substack{s \in \Lambda, t \notin \Lambda \\|s-t|=1}} U\left(\varphi_{s}-\varphi_{t}\right), \varphi \in \Phi_{\Lambda}^{n, k, i} .
$$

The Gibbs state $\left\langle>_{A, \beta}^{n, k, i}\right.$ in $\Lambda$ with hamiltonian $H$ and inverse temperature $\beta$ is defined by the formula

$$
\langle f\rangle_{\Lambda, \beta}^{n, k, i}=\operatorname{Tr}\left(f \exp \left\{-\beta H_{\Lambda}^{n, k, i}\right\}\right) / \operatorname{Tr}\left(\exp \left\{-\beta H_{\Lambda}^{n, k, i}\right\}\right) .
$$

Theorem. Let the conditions (i) and (ii) be satisfied. Then

1. the configurations $\Psi^{n, k, i}$, defined in (4), are the ground states of the hamiltonian $H$;

2. if $\beta>\beta(U, n)$, then the family of states \langle\rangle$_{\Lambda, \beta}^{n, k, i}$ is compact as $\Lambda \rightarrow \infty,(n, k, \beta, i$ are fixed) and if \langle\rangle$_{\beta}^{n, k, i}$ is any limit point of this family, then

$$
\left\langle\varphi_{t}\right\rangle^{n, k, i}=n t_{i}+k,
$$

and the probability of the event $B_{t}=\left\{\varphi: \varphi_{t} \neq \Psi_{t}^{n, k, i}\right\}$, calculated for the state \langle\rangle$_{\beta}^{n, k, i}$, tends to zero as $\beta \rightarrow \infty$.

\section{Proofs}

Let

$$
\Psi(s)=\Psi^{n, k, i}\left(s_{1}, s_{2}\right)=n s_{1}+k, \quad n, k \in \mathbb{Z}^{1}, \quad s=\left(s_{1}, s_{2}\right) \in \mathbb{Z}^{2}
$$

be a ground state which will be fixed throughout this section. We assume that $n>0$. Let $|\Lambda|<\infty, \Lambda \subset \mathbb{Z}^{2}$. By a configuration $\varphi$ in $\Lambda$ with boundary condition $\Psi$ we shall mean any mapping $\varphi: \mathbb{Z}^{2} \rightarrow \mathbb{Z}^{1}, \varphi(s)=\Psi(s)$ for $s \notin \Lambda$.

The proof is based on a suitable revised contour technique.

In order to define the set of contours of the configuration $\varphi$, we consider the difference $\tilde{\varphi}=\varphi-\Psi$. Let $\ell \in \mathbb{Z}^{1}$, and $A_{\ell}=\left\{s \in \mathbb{Z}^{2}, \tilde{\varphi}(s)>\ell\right\}$. (For $\ell<0$, the set $A_{\ell}$ is infinite.) The boundary $\partial A_{\ell}$ is defined as the set of all bonds $\gamma^{*} \in\left(\mathbb{Z}^{2}\right)^{*}$, which are dual to the bonds $\gamma=(s, t) \in \mathbb{Z}^{2}$ with the property: $s \in A_{\ell}, t \notin A_{\ell}$. The set $\partial A_{\ell}$ is split up into the sum of its connected components, $\partial A_{\ell}=\bigcup \Gamma_{i}$. Let us orient each component $\Gamma \subset \partial A_{\ell}$ in such a way that the corresponding connected component $B$ of $A_{\ell}$, whose boundary contains $\Gamma(\Gamma \subset \partial B)$ is situated on the left side of $\Gamma$. More precisely, for any $\gamma^{*} C \Gamma$ its orientation is chosen in such a way that the pair $\left(\gamma^{*},(\overrightarrow{t, s})\right)$ is left-oriented.

A set $\Gamma$ oriented in such a way is called an $\ell$-contour, or simply a contour. The union of all $\ell$-contours, $-\infty<\ell<\infty$ forms a set $\Gamma(\varphi)$ - the set of contours of the 
configuration $\varphi$. It should be noted that different contours in $\Gamma(\varphi)$ may intersect or even coincide, so the set $\Gamma(\varphi)$ is the set of contours with multiplicities.

The set of (oriented) contours $\mathscr{T}=\left\{\Gamma, \Gamma \subset \Lambda^{*} \subset \mathbb{Z}^{2 *}\right\}$ is called admissible if for all $\gamma^{*} \in \Gamma_{1} \cap \Gamma_{2}, \Gamma_{i} \in \mathscr{T}, i=1,2$, the two orientations of $\gamma^{*}$, induced by that of $\Gamma_{1}, \Gamma_{2}$, coincide. It can be seen immediately that for any $\varphi$ the set $\Gamma(\varphi)$ is admissible. Moreover, for any admissible set of contours $\mathscr{T}$ a configuration $\varphi(\mathscr{T})$ can be constructed in such a way that if $\mathscr{T}=\Gamma\left(\varphi^{\prime}\right)$ for some configuration $\varphi^{\prime}$, then $\varphi(\mathscr{T})=\varphi^{\prime}$.

In order to construct the configuration $\varphi(\mathscr{T})$, let us fix some point $s \notin \Lambda$, and for each $t \in \mathbb{Z}^{2}$ fix some oriented path $\ell_{t} \in \mathbb{Z}^{2}$ which starts at $s$ and terminates at $t$. Now the configuration $\varphi(\mathscr{T})$ is given by the formula $\varphi(t)=\Psi(t)+f\left(\ell_{t}\right)$, where

$$
f\left(\ell_{t}\right)=\sum_{\gamma \in \ell_{t}} f(\gamma)
$$

and

$$
f(\gamma)= \begin{cases}0, & \text { if } \gamma^{*} \notin \Gamma \text { for all } \Gamma \in \mathscr{T} \\ \left|\left\{\Gamma \in \mathscr{T}, \gamma^{*} \in \Gamma\right\}\right|, & \text { if the pair }\left(\gamma^{*}, \gamma\right) \text { is left-oriented } \\ -\left|\left\{\Gamma \in \mathscr{T}, \gamma^{*} \in \Gamma\right\}\right|, & \text { if the pair }\left(\gamma^{*}, \gamma\right) \text { is right-oriented }\end{cases}
$$

It is easy to see that the configuration $\varphi(\mathscr{T})$ is thus well-defined.

Now, let $\mathscr{T}$ be some admissible set of contours, $\Gamma \in \mathscr{T}$ and $\mathscr{T}^{\prime}=\mathscr{T} \backslash \Gamma$. (If the multiplicity of $\Gamma$ in $\mathscr{T}$ is more than 1 , then $\Gamma$ still belongs to $\mathscr{T}^{\prime}$.) For the set of configurations $C_{\Gamma}=\{\eta, \Gamma \in \Gamma(\eta)\}$, one can define the transformation $I_{\Gamma}$ given by the formula $I_{\Gamma}(\eta) \equiv \eta_{\Gamma}=\varphi(\Gamma(\eta) \backslash \Gamma)$. This is the analog of the Peierls transform. As usual, one is interested in the estimation of the energy difference $H(\varphi)-H\left(\varphi_{\Gamma}\right)$ for $\varphi \in C_{\Gamma}$ from below.

Lemma 1. For $\varphi \in C_{\Gamma}, H(\varphi)-H\left(\varphi_{\Gamma}\right) \geqq C_{n}|\Gamma|$, with $C_{n}=\frac{1}{2} \min \{(1 / 2)[U(r+1)$ $+U(r-1)]-U(r), r=1, \ldots, n, U(1)-U(0)\}>0$. Here $n$ is the same as in the ground state $\Psi$ (see (4)).

Proof. Let $\Gamma=\left\{\gamma^{*} ; \gamma^{*} \in\left(\mathbb{Z}^{2}\right)^{*}\right\}$. Consider the set $\Gamma^{*}=\left\{\gamma \in \mathbb{Z}^{2}, \gamma^{*} \in \Gamma\right\}$, and for any bond $\gamma \in \Gamma^{*}$, let $\gamma^{+}, \gamma^{-}$be its endpoints, and moreover let $\tilde{\varphi}\left(\gamma^{+}\right)>\tilde{\varphi}\left(\gamma^{-}\right)$. One sees immediately that

$$
\tilde{\varphi}\left(\gamma^{+}\right)-\tilde{\varphi}\left(\gamma^{-}\right)=\tilde{\varphi}_{\Gamma}\left(\gamma^{+}\right)-\tilde{\varphi}_{\Gamma}\left(\gamma^{-}\right)+1
$$

By the definition of $H$

$$
H(\varphi)-H\left(\varphi_{\Gamma}\right)=\sum_{\gamma \in \Gamma^{*}}\left[U\left(\varphi\left(\gamma^{+}\right)-\varphi\left(\gamma^{-}\right)\right)-U\left(\varphi_{\Gamma}\left(\gamma^{+}\right)-\varphi_{\Gamma}\left(\gamma^{-}\right)\right)\right]
$$

Let $\gamma_{1}^{ \pm}, \gamma_{2}^{ \pm}$be the first and second coordinates of the site $\gamma^{ \pm} \in \mathbb{Z}^{2}$. The bond $\gamma$ is called vertical if $\gamma_{1}^{-}=\gamma_{1}^{+}$and horizontal if $\gamma_{2}^{-}=\gamma_{2}^{+}$. We shall show first that each vertical bond contributes more than a fixed constant to the sum (8). Indeed, for a vertical bond

$$
\begin{gathered}
\varphi\left(\gamma^{+}\right)-\varphi\left(\gamma^{-}\right)=\tilde{\varphi}\left(\gamma^{+}\right)-\tilde{\varphi}\left(\gamma^{-}\right), \\
\varphi_{\Gamma}\left(\gamma^{+}\right)-\varphi_{\Gamma}\left(\gamma^{-}\right)=\tilde{\varphi}_{\Gamma}\left(\gamma^{+}\right)-\tilde{\varphi}_{\Gamma}\left(\gamma^{-}\right),
\end{gathered}
$$




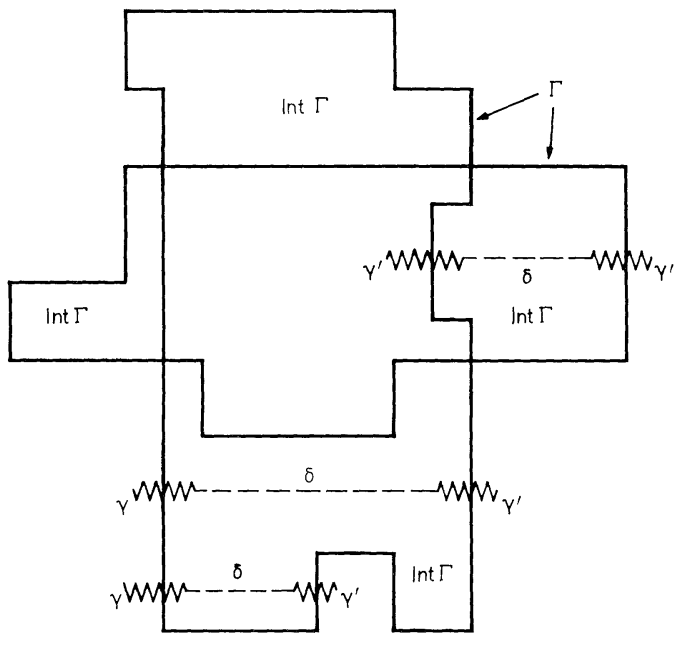

Fig. 1

hence

$$
\begin{aligned}
U\left(\varphi\left(\gamma^{+}\right)-\varphi\left(\gamma^{-}\right)\right)-U\left(\varphi_{\Gamma}\left(\gamma^{+}\right)-\varphi_{\Gamma}\left(\gamma^{-}\right)\right) & =U\left(\varphi\left(\gamma^{+}\right)-\varphi\left(\gamma^{-}\right)\right)-U\left(\varphi\left(\gamma^{+}\right)-\varphi\left(\gamma^{-}\right)-1\right) \\
& \geqq \min _{k>0}[U(k)-U(k-1)]=U(1)-U(0)
\end{aligned}
$$

by formula (7).

As for horizontal bonds, one sees immediately that the contribution of some of them to (8) is negative. We can circumvent this obstacle by splitting up the set of horizontal bonds into pairs.

To define this pairing, one first has to note that for any contour $\Gamma$ there is a uniquely defined configuration $\xi(\Gamma)$ such that $\Gamma$ is the only contour of $\xi(\Gamma)$, and its multiplicity is exactly one. Let $\operatorname{Int} \Gamma$ be the union of all one by one plaquettes of $\mathbb{R}^{2}$ centered at those vertices $s$ of $\mathbb{Z}^{2}$ for which $\xi(\Gamma)(s) \neq \Psi(s)$. Consider now the set $\mathscr{D}$ of all (horizontal) segments of the lines $\ell_{t} \subset \mathbb{Z}^{2}, \ell_{t}=\left\{(x, y) \in \mathbb{R}^{2}, y=t \in \mathbb{Z}^{1}, x \in \mathbb{R}^{1}\right\}$, such that $\delta \in \mathscr{D} \Leftrightarrow \delta=\ell_{t} \cap \operatorname{Int} \Gamma$ for some $t$. Now, two bonds $\gamma, \gamma^{\prime} \in \Gamma^{*}$ are paired if and only if there exists a $\delta \in \mathscr{D}$ such that both $\gamma \cap \delta, \gamma^{\prime} \cap \delta$ are non-empty (see Fig. 1).

We are going to show that each pair contributes at least some positive constant. Let two bonds $\gamma, \gamma^{\prime} \in \Gamma^{*}$ be paired. Then either $\gamma^{+}, \gamma^{+} \in \operatorname{Int} \Gamma$ or $\gamma^{-}, \gamma^{\prime} \in \operatorname{Int} \Gamma$. Consider the first possibility. Without loss of generality one may suppose that

$$
\begin{array}{ll}
\gamma_{1}^{-}=\gamma_{1}^{+}-1, & \gamma_{1}^{\prime}=\gamma_{1}^{\prime+}+1, \\
\gamma_{1}^{+}<\gamma_{1}^{\prime+}, & \gamma_{2}^{+}=\gamma_{2}^{-}=\gamma_{2}^{\prime+}=\gamma_{2}^{\prime-} .
\end{array}
$$

As for the Peierls transform

$$
\begin{array}{ll}
\varphi_{\Gamma}\left(\gamma^{-}\right)=\varphi\left(\gamma^{-}\right), & \varphi_{\Gamma}\left(\gamma^{\prime-}\right)=\varphi\left(\gamma^{\prime-}\right), \\
\varphi_{\Gamma}\left(\gamma^{+}\right)=\varphi\left(\gamma^{+}\right)-1, & \varphi_{\Gamma}\left(\gamma^{+}\right)=\varphi\left(\gamma^{+}\right)-1 .
\end{array}
$$




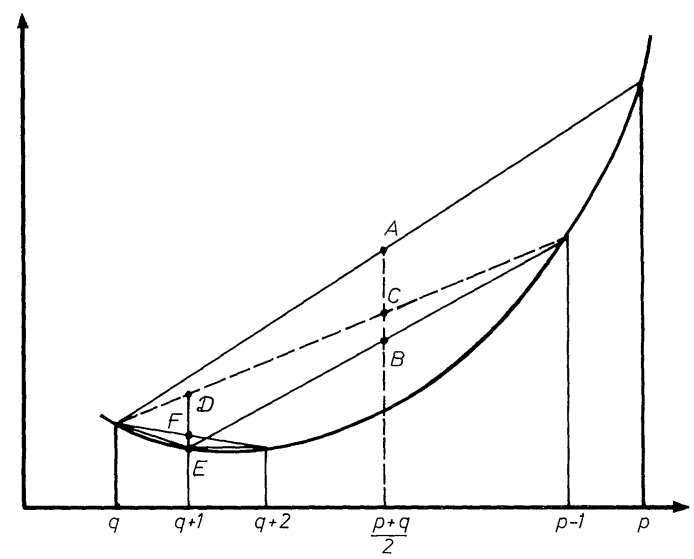

Fig. 2

Again there are two possibilities: either

$$
\tilde{\varphi}\left(\gamma^{+}\right)>\tilde{\varphi}\left(\gamma^{\prime-}\right)+n
$$

or

$$
0<\tilde{\varphi}\left(\gamma^{+}\right) \leqq \tilde{\varphi}\left(\gamma^{\prime-}\right)+n
$$

If (9a) holds, then $\varphi\left(\gamma^{+}\right)>\varphi\left(\gamma^{\prime-}\right)$, and again

$$
\begin{aligned}
U\left(\varphi\left(\gamma^{+}\right)-\varphi\left(\gamma^{\prime-}\right)\right) & -U\left(\varphi_{\Gamma}\left(\gamma^{+}\right)-\varphi_{\Gamma}\left(\gamma^{\prime-}\right)\right)=U\left(\varphi\left(\gamma^{+}\right)-\varphi\left(\gamma^{\prime-}\right)\right) \\
& -U\left(\varphi\left(\gamma^{++}\right)-\varphi\left(\gamma^{\prime-}\right)-1\right) \geqq U(1)-U(0)
\end{aligned}
$$

of course, $\varphi\left(\gamma^{+}\right)>\varphi\left(\gamma^{-}\right)+n$, and by the same reason

$$
U\left(\varphi\left(\gamma^{+}\right)-\varphi\left(\gamma^{-}\right)\right)-U\left(\varphi_{\Gamma}\left(\gamma^{+}\right)-\varphi_{\Gamma}\left(\gamma^{-}\right)\right)>U(1)-U(0) .
$$

So, in case (9a), each of the bonds $\gamma, \gamma^{\prime}$ contributes as before.

We are left with (9b) when the contribution of $\gamma^{\prime}$ is negative. Let $p=\varphi\left(\gamma^{+}\right)$ $-\varphi\left(\gamma^{-}\right), q=\varphi\left(\gamma^{\prime}\right)-\varphi\left(\gamma^{+}\right)$, then $0 \leqq q<n<p$ by definition of the contours. Now,

$$
\begin{aligned}
& U\left(\varphi\left(\gamma^{+}\right)-\varphi\left(\gamma^{-}\right)\right)+U\left(\varphi\left(\gamma^{\prime+}\right)-\varphi\left(\gamma^{\prime-}\right)\right)-\left[U\left(\varphi_{\Gamma}\left(\gamma^{+}\right)-\varphi_{\Gamma}\left(\gamma^{-}\right)\right)+U\left(\varphi_{\Gamma}\left(\gamma^{+}\right)-\varphi_{\Gamma}\left(\gamma^{\prime-}\right)\right)\right] \\
& \quad=U(p)+U(q)-[U(p-1)+U(q+1)] .
\end{aligned}
$$

Now we shall use the following simple

Lemma 2. If $U$ is convex, and $q<n<p$ are integers then

$$
U(p)+U(q)-[U(p-1)+U(q+1)] \geqq \frac{1}{2}[U(q)+U(q+2)]-U(q+1) .
$$

For the proof the reader is invited to inspect Fig. 2, where $U(p)+U(q)$ $-[U(p-1)+U(q+1)]=2 A B$, while $A B>C B, C B=(1 / 2) D E, D E \geqq F E=\frac{1}{2}[U(q)$ 
$+U(q+2)]-U(q+1)$. Thus the desired contribution is not less than

$$
\min _{r: 1 \leqq r \leqq n}\left\{\frac{1}{2}[U(r+1)+U(r-1)]-U(r)\right\},
$$

and Lemma 1 follows.

Now, let \langle\rangle$_{A, \beta}^{n, k, i}$ be the Gibbs state in the finite volume $\Lambda$, defined by the hamiltonian $H$ at inverse temperature $\beta$ and boundary condition $\Psi$. Then one has the following estimate of the probability $\operatorname{Pr}$ of a given contour $\Gamma$ to occur in the state \langle\rangle$_{\Lambda, \beta}^{n, k, i}$ :

$\operatorname{Pr}\{\varphi: \Gamma \in \Gamma(\varphi), \varphi(s)=\Psi(s), s \notin \Lambda\}$,

$$
\begin{aligned}
& =\sum_{\varphi, \Gamma \in \Gamma(\varphi)} \exp \{-\beta H(\varphi)\} / \sum_{\varphi} \exp \{-\beta H(\varphi)\} \\
& \leqq \exp \left\{-\beta C_{n}|\Gamma|\right\} \frac{\sum_{\varphi, \Gamma \in \Gamma(\varphi)} \exp \{-\beta H(\varphi)\}+\sum_{\begin{array}{c}
\varphi, \Gamma \notin \Gamma(\varphi) \\
\Gamma \text { compatible with } \Gamma(\varphi)
\end{array}} \exp \{-\beta H(\varphi)\}}{\sum_{\varphi} \exp \{-\beta H(\varphi)\}} \\
& \leqq \exp \left\{-\beta C_{n}|\dot{\Gamma}|\right\} .
\end{aligned}
$$

The second sum is taken over those configurations $\varphi$ for which the family $\Gamma \cup \Gamma(\varphi)$ is admissible. The estimate (10) is known as the Peierls estimate. From (10) it follows in a standard manner that for any $t \in \Lambda$ and large enough $\beta \operatorname{Pr}\{\varphi: \mid \varphi(t)$ $-\Psi(t) \mid \geqq 1\} \leqq e^{-\beta d_{n}}$ for some $d_{n}=d_{n}\left(C_{n}\right)>0$, and moreover,

$$
\operatorname{Pr}\{\varphi:|\varphi(t)-\Psi(t)| \geqq r\} \leqq e^{-\beta r d_{n}} .
$$

Indeed, if $|\varphi(t)-\Psi(t)| \geqq r$ then there are at least $r$ contours in $\Gamma(\varphi)$ surrounding $t$.

From (11) the compactness of the family of states \langle\rangle$_{\Lambda, \beta}^{n, k, i}, \Lambda \subset \mathbb{Z}^{2},|\Lambda|<\infty$ follows easily.

Let \langle\rangle$_{\beta}^{n, k, i}$ be some limit point of the set \langle\rangle$_{A, \beta}^{n, k, i}$. The statement (5) of the theorem follows from the fact that for all $\Lambda$ the state \langle\rangle$_{A, \beta}^{n, k, i}$ is symmetric under the reflection $\varphi \rightarrow \bar{\varphi}$ with $\bar{\varphi}(s+t)=\Psi(t)-\varphi(t-s)$. The rest of the theorem follows from the Peierls estimate.

\section{Discussion}

1. We have shown that the "stair" ground state $\Psi\left(s_{1}, s_{2}\right)=n s_{1}$ is stable at non-zero temperature. It seems that other choices of boundary conditions - i.e., non-linear or linear with a non-integer factor - do not give any new Gibbs states which are not mixtures of the already constructed ones.

2. In their very deep and interesting paper [4], Fröhlich and Spencer were able to show that in certain integer-valued spin models in two dimensions a sort of roughening transition takes place, which manifests itself by passing from finite to logarithmically divergent fluctuations as temperature increases. Because of a more complicated structure of stable ground states in our models, a natural question arises: How many roughening transitions may occur in them? A priori it is possible that the critical temperature for a given state \langle\rangle$^{n, k, i}$ can depend on $n$. But we were unable to prove any result of this type. 


\section{References}

1. Gallavotti, G.: The phase separation line in the two-dimensional Ising model. Commun. Math. Phys. 27, 103 (1972)

2. Aizenman, M.: Translation invariance and instability of phase coexistence in the two-dimensional Ising system. Commun. Math. Phys. 73, 83 (1981)

3. Sinai, Ya.: The theory of phase transitions. Moscow: Nauka 1980

4. Fröhlich, J., Spencer, T.: The Kosterlitz-Thouless transition in two-dimensional abelian spin systems and the Coulomb gas. Commun. Math. Phys. 81, 527 (1981)

Communicated by Ya. G. Sinai

Received June 1, 1982 